\title{
APPROXIMATE SELECTIONS AND FIXED POINTS FOR UPPER SEMICONTINUOUS MAPS WITH DECOMPOSABLE VALUES
}

\author{
ARRIGO CELLINA, GIOVANNI COLOMBO AND ALESSANDRO FONDA
}

\begin{abstract}
We prove the existence of continuous approximate selections of upper semicontinuous maps from a separable locally compact metric space $S$ into the decomposable subsets of $L^{1}(T, Z)$. We then extend a fixed point theorem of Kakutani to upper semicontinuous maps with decomposable values.
\end{abstract}

1. Introduction. In [1], Antosiewicz and Cellina presented an analogue of Michael's selection theorem for a special multivalued function related to differential inclusions: the image of a point was the set of selections of a given multivalued map. The main tool in that proof was the idea of piecing together a finite number of measurable functions. In [6], Hiai and Umegaki, modifying somewhat an earlier definition of Rockafellar [8], called any set which is closed with respect to the operation of "piecing" decomposable. A subset $F$ of $L^{1}(I)$ is decomposable whenever, given $u$ and $v$ in $F$ and any measurable partition of $I$ into $A$ and $B, u \chi_{A}+v \chi_{B}$ still belongs to $F$. In the same paper they proved that a closed set is decomposable if and only if it is the set of measurable selections of a multifunction. However, we shall retain the word decomposable for the sake of simplicity.

In [2], it was shown that the set of selections of a constant multivalued map has the fixed point property; in [4] the existence of a fixed point was proven for a self-map of a decomposable set.

The purpose of the present note is first to present a theorem on the existence of an approximate selection to an upper semicontinuous multifunction with decomposable values, and then to obtain a further extension of the above fixed point theorem on decomposable sets to cover the case of upper semicontinuous maps with decomposable values. This result is the analogue of Kakutani's theorem with convexity replaced by decomposability.

2. Notation and basic definitions. In what follows, $S$ is a metric space with distance $d ; T$ is a compact topological space with a $\sigma$-field $\mathcal{M}$ of measurable subsets of $T$ given by a nonnegative, finite nonatomic measure $\mu_{0} ; Z$ is a separable Banach space with the norm $|\cdot| ; L^{1}(T, Z)$ is the Banach space of functions $u: T \rightarrow Z$, integrable in the Bochner sense, with the norm $\|u\|_{1}=\int_{T}|u(t)| d \mu_{0}$ and the distance $d_{1}(u, v)=\|u-v\|_{1}$. In the product space $S \times L^{1}$ the distance will be the sum of $d$ and $d_{1}$. The $L^{1}$ distance of $u$ from a set $F$ is $d_{1}(u, F)=\inf _{v \in F}\|u-v\|_{1}$.

A set $H \subseteq L^{1}(T, Z)$ is called decomposable if $u \cdot \chi_{A}+v \cdot \chi_{T \backslash A} \in H$ for every $u, v \in H$ and $A \in \mathcal{M} ; \operatorname{dec}(\mathcal{K})$ is the set of all decomposable subsets of $\mathcal{K} \subseteq L^{1}(T, Z)$.

Upper semicontinuity is meant in the usual $(\varepsilon, \delta)$ sense.

Received by the editors September 16, 1985.

1980 Mathematics Subject Classification (1985 Revision). Primary 54C65, 28B20, 54H25. 
In a metric space, $B(K, \delta)$ is the open $\delta$-neighborhood of a set $K$. The graph of a map $F$ is denoted by $\operatorname{gr}\{F\}$. A function $f_{\varepsilon}$ is an $\varepsilon$-approximate selection of a map $F$ if

$$
\operatorname{gr}\left\{f_{\varepsilon}\right\} \subseteq B(\operatorname{gr}\{F\}, \varepsilon)
$$

3. Main results. In this section we prove an approximate selection theorem, and we use this result to prove a fixed point theorem for upper semicontinuous multivalued functions.

THEOREM 1. Let $S$ be a locally compact and separable metric space, and $F: S \rightarrow$ $\operatorname{dec}\left(L^{1}(T, Z)\right)$ an upper semicontinuous map. Then for every $\varepsilon>0$ there exists a continuous $\varepsilon$-approximate selection of $F$. Moreover, when $F(S)$ is decomposable, $f_{\varepsilon}(S) \subseteq F(S)$.

Proof. The proof is divided into two steps.

STEP 1. We show that the theorem is true when $S$ is compact. Fix $\varepsilon>0$; by the upper semicontinuity of $F$, for each $s \in S$ there is a $\delta(s)>0$ such that $F\left(s^{\prime}\right) \subseteq B(F(s), \varepsilon / 3)$ whenever $s^{\prime} \in B(s, \delta(s))$. We can choose $\delta(s)<\varepsilon / 3$. Since $S$ is compact and $\{B(s, \delta(s) / 2): s \in S\}$ is an open covering of $S$, there exist $s_{1}, \ldots, s_{n} \in S$ such that, setting $\delta_{i}=\delta\left(s_{i}\right) / 2$, the balls $B\left(s_{i}, \delta_{i}\right)(i=1, \ldots, n)$ form a finite subcovering of $S$. Let $\left\{p_{i}: i=1, \ldots, n\right\}$ be a continuous partition of unity subordinate to it, and choose arbitrarily $u_{i} \in F\left(s_{i}\right)(i=1, \ldots, n)$. We shall construct $f_{\varepsilon}(s)$ as an appropriate decomposition of these maps.

Let us choose $v_{i j} \in F\left(s_{j}\right)(i, j=1, \ldots, n)$ such that

$$
\begin{aligned}
d_{1}\left(u_{i}, v_{i j}\right) & =\int_{T}\left|u_{i}(t)-v_{i j}(t)\right| d \mu_{0} \\
& \leq \inf _{v \in F\left(s_{j}\right)} \int_{T}\left|u_{i}(t)-v(t)\right| d \mu_{0}+\frac{\varepsilon}{3}=d_{1}\left(u_{i}, F\left(s_{j}\right)\right)+\frac{\varepsilon}{3}
\end{aligned}
$$

and define the set functions

$$
\mu_{i j}(E)=\int_{E}\left|u_{i}(t)-v_{i j}(t)\right| d \mu_{0} \quad(i, j=1, \ldots, n)
$$

for every $E \in \mathcal{M}$. It is easy to see that, for each $i, j \in\{1, \ldots, n\}, \mu_{i j}$ is a finite nonatomic measure over $\mathcal{M}$. Following an idea of Fryszkowski [3] (see also [7]) we apply here a consequence of Lyapunov's theorem (cf. [5]): there exists a family $\left(A_{\alpha}\right)_{\alpha \in[0,1]}$ of measurable subsets of $T$ such that

(P $A_{\alpha} \subseteq A_{\beta}$ if $\alpha \leq \beta$,

$\left(\mathrm{P}_{2}\right) \mu_{i j}\left(A_{\alpha}\right)=\alpha \mu_{i j}(T)(i, j=1, \ldots, n)$,

$\left(\mathrm{P}_{3}\right) \mu_{0}\left(A_{\alpha}\right)=\alpha \mu_{0}(T)$.

Set $\alpha_{0} \equiv 0, \alpha_{i}(s)=p_{1}(s)+\cdots+p_{i}(s)$ and define the approximate selection as

$$
f_{\varepsilon}(s)=\sum_{i=1}^{n} u_{i} \chi_{A_{\alpha_{i}(s)} \backslash A_{\alpha_{i-1}(s)}}
$$


We claim that $f_{\varepsilon}$ has the required properties. First of all $f_{\varepsilon}$ is continuous. In fact, fix $s_{0}$; then

$$
\begin{aligned}
& \left\|f_{\varepsilon}(s)-f_{\varepsilon}\left(s_{0}\right)\right\|_{1}=\| \sum_{i=1}^{n} u_{i}\left(\chi_{A_{\alpha_{i}(s)} \backslash A_{\alpha_{i-1}(s)}}-\chi_{\left.A_{\alpha_{i}\left(s_{0}\right)} \backslash A_{\alpha_{i-1}\left(s_{0}\right)}\right)} \|_{1}\right. \\
& \leq \sum \int_{T}\left|u_{i}(t)\right| \cdot\left|\chi_{A_{\alpha_{i}(s)} \backslash A_{\alpha_{i-1}(s)}}(t)-\chi_{A_{\alpha_{i}\left(s_{0}\right)} \backslash A_{\alpha_{i-1}\left(s_{0}\right)}}(t)\right| d \mu_{0} \\
& \leq \sum \int_{T}\left|u_{i}\right|\left\{\left|\chi_{A_{\alpha_{i}(s)}}-\chi_{A_{\alpha_{i}\left(s_{0}\right)}}\right|+\left|\chi_{A_{\alpha_{i-1}(s)}}-\chi_{A_{\alpha_{i-1}\left(s_{0}\right)}}\right|\right\} d \mu_{0} \\
& =\sum\left\{\int_{A_{\alpha_{i}(s)} \Delta A_{\alpha_{i}\left(s_{0}\right)}}\left|u_{i}\right| d \mu_{0}+\int_{A_{\alpha_{i-1}(s)} \Delta A_{\alpha_{i-1}\left(s_{0}\right)}}\left|u_{i}\right| d \mu_{0}\right\},
\end{aligned}
$$

and, by the integrability of $u_{i}$ and $\left(\mathrm{P}_{3}\right)$, the continuity of $f_{\varepsilon}$ follows.

It is clear that if $F(S)$ is decomposable then $f_{\varepsilon}(S) \subseteq F(S)$. It remains to verify that $f_{\varepsilon}$ is an $\varepsilon$-approximate selection of $F$. For this purpose, fix $s \in S$ and let $I(s)=\left\{i \in\{1, \ldots, n\}: p_{i}(s)>0\right\}$ and $\bar{i} \in I(s)$ such that $\delta_{i}=\max \left\{\delta_{i}: i \in I(s)\right\}$.

Then, for every $i \in I(s)$, we have $s_{i} \in B\left(s_{\bar{i}}, 2 \delta_{\bar{i}}\right)$ so that $F\left(s_{i}\right) \subseteq B\left(F\left(s_{\bar{i}}\right), \varepsilon / 3\right)$ and

$$
\mu_{i \bar{i}}(T) \leq d_{1}\left(u_{i}, F\left(s_{i}^{-}\right)\right)+\varepsilon / 3 \leq \frac{2}{3} \varepsilon
$$

Moreover, since $F\left(s_{\bar{i}}\right)$ is decomposable, we have that

$$
\omega_{i} \equiv \sum_{i \in I(s)} v_{i \bar{i}} \cdot \chi_{A_{\alpha_{i}(s)} \backslash A_{\alpha_{i-1}(s)}} \in F\left(s_{\bar{i}}^{\bar{r}}\right) .
$$

Finally,

$$
\begin{aligned}
d\left(\left(s, f_{\varepsilon}(s)\right),\left(s_{\bar{i}}^{\bar{i}}, \omega_{\bar{i}}\right)\right) & \leq d\left(s, s_{\bar{i}}\right)+\left\|\sum_{i \in I(s)}\left(u_{i}-v_{i \bar{i}}\right) \chi_{A_{\alpha_{i}(s)} \backslash A_{\alpha_{i-1}(s)}}\right\|_{1} \\
& \leq \delta(s)+\sum_{i \in I(s)} \|\left(u_{i}-v_{i \bar{i}}\right) \chi_{A_{\alpha_{i}(s)} \backslash A_{\alpha_{i-1}(s)} \|_{1}} \\
& \leq \frac{\varepsilon}{3}+\sum_{i \in I(s)} \mu_{i \bar{i}}\left(A_{\alpha_{i}(s)} \backslash A_{\alpha_{i-1}(s)}\right) \\
& =\frac{\varepsilon}{3}+\sum_{i \in I(s)}\left[\alpha_{i}(s)-\alpha_{i-1}(s)\right] \mu_{i \bar{i}}(T) \\
& \leq \frac{\varepsilon}{3}+\frac{2}{3} \varepsilon \sum_{i \in I(s)} p_{i}(s)=\varepsilon
\end{aligned}
$$

and the proof of the first step is complete.

STEP 2 . Let $S$ be locally compact and separable. Then there exists a sequence $\left(\Omega_{n}\right)_{n}$ of open sets such that $\bar{\Omega}_{n}$ is compact nonempty, $\bar{\Omega}_{n} \subseteq \Omega_{n+1}$ and $\bigcup_{n} \Omega_{n}=S$.

Fix $\varepsilon>0$; by the upper semicontinuity of $F$, for each $s \in S$ there is $\delta(s)>0$ such that $F\left(s^{\prime}\right) \subseteq B(F(s), \varepsilon / 9)$ whenever $s^{\prime} \in B(s, \delta(s))$. We may choose $\delta(s)<\varepsilon / 3$. The hypothesis on $S$ allows us to consider a countable number of balls $B\left(s_{i}, \delta_{i}\right)$, with $\delta_{i}=\delta\left(s_{i}\right) / 2$, which cover $S$, such that for every $n \in \mathbf{N}$ the set $I_{n}=\{i \in$ $\left.\mathbf{N}: B\left(s_{i}, \delta_{i}\right) \cap \bar{\Omega}_{n} \neq \varnothing\right\}$ is finite. Let $\left\{p_{i}: i \in \mathbf{N}\right\}$ be a continuous partition of unity subordinate to this covering of $S$. In order to construct $f_{\varepsilon}$, choose arbitrarily 
$u_{i} \in F\left(s_{i}\right)(i \in \mathbf{N})$. As it has been shown in Step 1 , we can construct on every $\bar{\Omega}_{n}$ an $\varepsilon$-approximate selection $f_{\varepsilon}^{(n)}$, and for every $s \in S$ there is $\omega_{i}^{(n)}$ (depending on $s)$ in $F\left(s_{\bar{i}}\right)$ such that

$$
d\left(\left(s, f_{\varepsilon}^{(n)}(s)\right),\left(s_{\bar{i}}, \omega_{\bar{i}}^{(n)}\right)\right)<\varepsilon / 3,
$$

where $\bar{i}$ satisfies $\delta_{i}=\max \left\{\delta_{i}: p_{i}(s)>0\right\}$.

Consider a family $\left(C_{\alpha}\right)_{\alpha \in[0,1]}$ of measurable subsets of $T$ such that

(1) $C_{\alpha} \subseteq C_{\beta}$ if $\alpha \leq \beta$,

(2) $\mu_{0}\left(C_{\alpha}\right)=\alpha \mu_{0}(T)(\alpha \in[0,1])$.

Set $f_{\varepsilon}(s)=f_{\varepsilon}^{(2)}(s)$ for $s$ in $\bar{\Omega}_{1}$; in general, let $n \geq 2$ be such that $s \in \bar{\Omega}_{n} \backslash \bar{\Omega}_{n-1}$. Set

$$
\alpha(s)=d\left(s, \Omega_{n-1}\right) /\left(d\left(s, \Omega_{n-1}\right)+d\left(s, S \backslash \Omega_{n}\right)\right)
$$

and define

$$
f_{\varepsilon}(s)=f_{\varepsilon}^{(n+1)}(s) \chi_{C_{\alpha(s)}}+f_{\varepsilon}^{(n)}(s) \chi_{T \backslash C_{\alpha(s)}} ;
$$

$f_{\varepsilon}$ is well defined and continuous on $S$. Moreover, setting

$$
\omega_{\bar{i}}=\omega_{\bar{i}}^{(n+1)} \chi_{C_{\alpha(s)}}+\omega_{\bar{i}}^{(n)} \chi_{T \backslash C_{\alpha(s)}}
$$

we have that $\omega_{\bar{i}} \in F\left(s_{\bar{i}}^{-}\right)$and, finally, $d\left(\left(s, f_{\varepsilon}(s)\right),\left(s_{\bar{i}}, \omega_{\bar{i}}^{-}\right)\right) \leq \varepsilon$.

The following is a fixed point theorem for upper semicontinuous multivalued maps.

THEOREM 2. Let $K$ be a nonempty closed subset of $L^{1}(T, Z)$, and $F: K \rightarrow$ $\operatorname{dec}(\mathcal{K})$ an upper semicontinuous map with closed graph. Moreover, let $F(\mathcal{K})$ be decomposable and totally bounded. Then there exists $\bar{s} \in \mathcal{K}$ with $\bar{s} \in F(\bar{s})$.

Proof. We can apply Theorem 1 to the map $G=\left.F\right|_{\overline{F(\mathcal{K})}}$ : For each $n \in \mathbf{N}$ there exists $g_{n}: \overline{F(K)} \rightarrow F(\mathcal{K})$ which is a $1 / n$-approximate selection of $G$. Then $g_{n}$ satisfies the assumptions of Fryszkowski's fixed point theorem [4], so that there exists $s_{n}=g_{n}\left(s_{n}\right)$, for every $n \in \mathbf{N}$. By the compactness of $\overline{F(K)}$ we can assume $\left(s_{n}\right)_{n}$ to converge to some point $\bar{s}$, which is a fixed point of $G$.

\section{REFERENCES}

1. H. A. Antosiewicz and A. Cellina, Continuous selections and differential relations, J. Differential Equations 19 (1975), 386-398.

2. A. Cellina, A fixed point theorem for subsets of $L^{1}$, Cahiers Ceremade 8205 (1982).

3. A. Fryszkowski, Continuous selections for a class of non-convex multivalued maps, Studia Math. 76 (1983), 163-174.

4. 215.

5. P. Halmos, The range of a vector measure, Bull. Amer. Math. Soc. 54 (1948), 417-421.

6. F. Hiai and H. Umegaki, Integrals, conditional expectations, and martingales of multivalued functions, J. Multivariate Anal. 7 (1977), 149-182.

7. C. Olech, Decomposability as a substitute for convexity, Lecture Notes in Math., Vol. 1091, Springer-Verlag, Berlin and New York, 1984, pp. 193-205.

8. R. T. Rockafellar, Integrals which are convex functions, Pacific J. Math. 24 (1968), 525539.

DEPARTMENT OF MATHEMATICS, INTERNATIONAL SCHOOL FOR ADVANCED STUdies (SISSA), TRIESTE, ITALY 